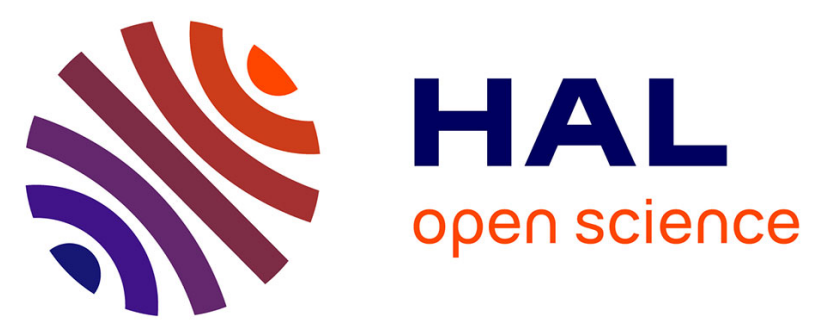

\title{
Le critère de la grande vitesse dans la planification des infrastructures ferroviaires : vers un changement de référentiel ? Une lecture normande
}

Jean Debrie, Anne-lise Guérin

\section{To cite this version:}

Jean Debrie, Anne-lise Guérin. Le critère de la grande vitesse dans la planification des infrastructures ferroviaires : vers un changement de référentiel ? Une lecture normande. RTS - Recherche Transports Sécurité, 2015, Usages de la vitesse, 2015 (01), pp.25-33. 10.4074/S076189801500103X . hal-01670604

\section{HAL Id: hal-01670604 \\ https://hal.science/hal-01670604}

Submitted on 21 Dec 2017

HAL is a multi-disciplinary open access archive for the deposit and dissemination of scientific research documents, whether they are published or not. The documents may come from teaching and research institutions in France or abroad, or from public or private research centers.
L'archive ouverte pluridisciplinaire HAL, est destinée au dépôt et à la diffusion de documents scientifiques de niveau recherche, publiés ou non, émanant des établissements d'enseignement et de recherche français ou étrangers, des laboratoires publics ou privés. 


\title{
Le critère de la grande vitesse dans la planification des infrastructures ferroviaires : vers un changement de référentiel ? Une lecture normande
}

\author{
High-speed rail in France : toward a new infrastructure approach ? \\ Lessons to be learnt from the "Ligne Nouvelle Paris Normandie" case
}

\author{
Jean Debrie · Anne-Lise Guérin \\ C IFSTTAR et Éditions NecPlus 2015
}

\begin{abstract}
Résumé Le développement des Lignes ferroviaires à grande vitesse (LGV) en France a constitué un outil majeur des politiques d'aménagement du territoire ces trente dernières années. Il traduit une lecture spécifique du rapport vitesse/territoire partagée par l'État, ses acteurs ferroviaires (SNCF, RFF) et les collectivités territoriales. Sans minorer la conflictualité induite par ces grands projets et leurs tracés, il reste qu'une vision partagée de la nécessité de cette grande vitesse ferroviaire a autorisé ce développement et les financements nécessaires. Un changement de période récent est pourtant à signaler. Ce changement est lié à deux facteurs, la difficulté de financement dans un contexte de contraction des ressources dédiées aux infrastructures nouvelles et la prise de conscience récente de la dégradation du réseau existant. Cette période voit alors émerger une remise en cause du tout TGV et une réflexion nouvelle sur la vitesse ferroviaire. Cette remise en cause, encore partielle, implique néanmoins un travail d'évolution du référentiel d'évaluation de l'infrastructure ferroviaire et une négociation entre le gestionnaire d'infrastructure et les collectivités territoriales. L'objectif de notre article, développé autour de l'exemple de la Ligne nouvelle Paris-Normandie et du débat public associé, est d'apprécier cette mobilisation du critère de la vitesse dans les exercices de planification et de prospectives territoriales.
\end{abstract}

Mots clés grande vitesse ferroviaire $\cdot$ planification $\cdot$ débat public $\cdot$ infrastructures

\footnotetext{
Jean Debrie $(\bowtie)$

Université Paris 1 Panthéon-Sorbonne

UMR Géographie-cités

13 rue du Four, 75006 Paris

e-mail : jean.debrie@univ-paris1.fr

Anne-Lise Guérin $(\bowtie)$

UMR Géographie-Cités

13 rue du Four, 75006 Paris

e-mail : annelizguerin@gmail.com
}

\begin{abstract}
The development of high-speed trains has been an important tool for national and regional planning in France for 30 years. Different levels of public policies (State, regional and local authorities) have funded a network linking a part of French metropolitan system. Analysis of planning documents can demonstrate a French political consensus on the importance of the High Speed Rail. But a change in the consensus seems to be emerging. The inability to finance all new railway projects and the need to finance the renovation of the existing network, require a new infrastructure approach. This approach is based on the construction of a technical model, la Ligne nouvelle, currently being negotiated between the actors of public policies and the manager of the national infrastructure. The purpose of this paper is to analyze this passage from the TGV to the Ligne nouvelle. This analysis is applied to a particular example, la Ligne nouvelle Paris Normandie, to illustrate this transition imagined to anticipate the evolution of high-speed rail networks in France.
\end{abstract}

Keywords High-speed rail · planning · infrastructure · public debate

\section{Introduction.}

\section{Grande vitesse ferroviaire et aménagement du territoire, une lecture spécifique de l'accessibilité}

Du schéma directeur des lignes à grande vitesse de 1991 au Schéma national des infrastructures de Transport issu des lois du Grenelle de l'environnement (SNIT) de 2011, la généralisation des lignes à grande vitesse a constitué un objectif partagé des collectivités territoriales, de l'État et de ses acteurs publics ferroviaires. La grande vitesse ferroviaire est à ce titre l'instrument principal d'une prospective d'un territoire national identifié comme système métropolitain. L'image d'un Train à grande vitesse (TGV) « grand RER d'une métropole France » pour reprendre la formule de Pierre Veltz [1] résume cette lecture repérable dans la plupart des documents nationaux et régionaux. Dans 
ces documents, la vitesse résume le critère d'accessibilité mesuré et évalué par ces gains de temps. Ce critère absolu de la vitesse semble pourtant récemment remis en cause dans un double contexte de hiérarchisation des investissements publics dédiés aux infrastructures et de dégradation du réseau existant. Les récents arbitrages de la commission 21 (juin 2013), en charge de la hiérarchisation du Schéma national des infrastructures de transport, témoignent d'un arrêt clair de la généralisation de la grande vitesse au profit d'une lecture plus complexe de l'accessibilité territoriale. Cet arbitrage politique s'inscrit plus globalement dans une remise en cause de l'effet TGV émanant du milieu académique puis progressivement reprise dans les milieux institutionnels. L'objectif de cet article est de proposer une lecture de cette évolution et de cette mobilisation du critère de la vitesse ferroviaire dans les politiques d'aménagement. L'analyse d'un espace spécifique - l'espace normand délimité dans cette approche par les deux territoires institutionnels régionaux (Haute-Normandie, Basse Normandie) - fournira les matériaux pour construire cette lecture.

Cette question de la vitesse ferroviaire est d'abord connue par l'objet technique qui lui donne corps. En France, on distingue ainsi le train classique (Train Express Régional (TER) et Intercité), dont la vitesse maximale de circulation oscille entre $100 \mathrm{~km} / \mathrm{h}$ et $220 \mathrm{~km} / \mathrm{h}$ pour des lignes non dégradées, et le TGV qui roule entre $300 \mathrm{~km} / \mathrm{h}$ et $320 \mathrm{~km} / \mathrm{h}$ dont les caractéristiques techniques permettent des relations rapides entre Paris et les grandes métropoles régionales et européennes. Une Ligne grande vitesse (LGV) en France est ainsi un dispositif technique spécifique qui autorise une circulation de trains dits TGV. Si ces trains peuvent circuler sur le réseau classique, ils ne peuvent atteindre la vitesse de $320 \mathrm{~km} / \mathrm{h}$ que sur des lignes dédiées et conçues spécifiquement pour leur circulation. Stéphane Dubois dans un article récent [2] met à jour cette transition d'une France ferroviaire pré-TGV à la mise en place d'une grande vitesse et d'un réseau associé engendrant un dualisme ferroviaire largement admis $[3 ; 4]$. Ce dualisme se traduit par un manque d'investissement chronique pour l'entretien du réseau classique au profit d'une mobilisation financière pour le développement du réseau à grande vitesse. La dégradation de l'état du réseau classique, malgré l'impulsion donnée au développement du transport régional par les régions depuis le transfert de compétence en 2002 est largement évaluée [5]. Et la priorité engagée sur les lignes à grande vitesse autorise la circulation de trains rapides, aux capacités de transport fortes, avec des fréquences de rotation et des taux de régularité élevés. Dès lors, la structuration $\mathrm{du}$ réseau ferroviaire à grande vitesse en radiale vers Paris participe d'une accessibilité ferroviaire différenciée. Cela s'est ajouté à l'appropriation de l'idée d' « enclavement » des territoires corrélée à la définition d'objectifs politiques de « désenclavement»: on définit des catégories de territoires plus ou moins attractifs en fonction notamment de l'idéal d' « accessibilité », dont la grande vitesse est l'outil principal.

Les discussions politiques autour des projets de grande vitesse force illustrent le constat du paradoxe entre réalité objective et imaginaire sociopolitique. Qu'est réellement cette grande vitesse pour les territoires? La compréhension du réel a déjà fait l'objet de nombreux écrits, traitant des effets objectivables et controversés de la grande vitesse, qu'ils soient territoriaux ou socio-économiques $[6 ; 7 ; 8]$. La relation de causalité entre attractivité/développement des territoires et nouvelle accessibilité ferroviaire est discutée dans la littérature grise comme dans les débats publics. La compréhension des représentations collectives et politiques de la vitesse ferroviaire, qui influe sur la production de l'espace, pose également de nombreuses questions. La grande vitesse, ses effets socio-économiques identifiables (ou non), est un puissant référentiel dans les politiques d'aménagement. L'objectif de cet article est de contribuer à l'analyse de ce référentiel et de son évolution actuelle. Il importe alors de préciser que la notion de référentiel ne renvoie pas directement dans notre approche à son acception politiste, utilisée d'ailleurs pour relire finement certaines questions de mobilité $[9 ; 10]$, mais bien au lien entre modèle d'évaluation technique d'une infrastructure de transport (le référentiel technique TGV) et conséquence de ce modèle sur les exercices de planification et de prospective territoriales. Sans rentrer dans une description technique de ce référentiel TGV porté par la SNCF et négocié avec Réseau Ferré de France (RFF) pour la création de nouvelles lignes, il importe ici de signaler qu'à cette vitesse des $300-320 \mathrm{~km} / \mathrm{h}$ correspond un ensemble de normes pour les lignes (rayons de courbure, pentes autorisées, signalétique, installation électrique, voies, quais, gares) et pour le matériel roulant (train, alimentation). Ce sont ces normes qui définissent le design (pour reprendre l'expression ferroviaire) et le coût d'une ligne grande vitesse.

Entre modèle technique et utilisation discursive, la grande vitesse apparaît alors comme un référentiel à décrypter de l'aménagement des territoires. À gros traits, qui seront précisés par l'analyse de l'espace normand, on peut observer un changement de période récent dans cette mobilisation du critère de la grande vitesse. L'engouement pour la grande vitesse, symbole de modernité et d'attractivité (traduisant l'idéal territorial des acteurs dans un contexte de mise en concurrence des territoires), est généralisé sur la période 1980-2010 pour la plupart des collectivités territoriales. Et l'adéquation entre cette grande vitesse et le besoin d'accessibilité des territoires n'est pas toujours centrale pour expliquer cet engouement des territoires potentiellement desservis. Philippe Subra [11], en conceptualisant une géopolitique propre à l'aménagement du territoire et aux réseaux de transports, en a étudié les ressorts. La grande 
vitesse acquiert un statut d'objet de conquête, ce qui entraîne des rivalités territoriales, des luttes d'influences jusqu'au déraisonnement politique dont les conséquences sur la production de l'espace sont réelles. Pour autant, un changement de période semble émerger, validé par le rapport de la commission 21 qui invite les acteurs publics à renouveler leur grille de lecture du rapport infrastructures de transport/territoires. La fin du « tout TGV » (pour reprendre la formule reprise et commentée dans la presse régionale et nationale au lendemain de la parution du rapport de la commission 21) est aujourd'hui débattue. Le débat est complexe, et à la hiérarchisation des projets de lignes à grande vitesse opérée par la commission (la plupart des projets étant renvoyés à une phase 2030 plus lointaine) succède bien sûr un jeu (géo) politique où chaque collectivité cherche à réinscrire sa desserte dans les priorités de financement. Pour autant, l'équation nouvelle (contraction à venir des fonds publics/dégradation du réseau existant) impose une évolution de la lecture de l'accessibilité ferroviaire. Les travaux de la commission 21 tout comme le Grand plan de modernisation du réseau (GPMR) porté par RFF signalent cette réorientation stratégique. Même si les ambitions ont été largement revues à la baisse, les projets de lignes à grande vitesse restent nombreux. Désormais, le maintien ou la remise à niveau, la modernisation voire le développement du réseau classique font aussi partie des priorités. Mais dans cette évolution, c'est surtout une idée émergente, le cran de vitesse manquant, qui doit être signalée. Elle marque une lecture nouvelle de la vitesse, encore partielle mais introduite sur quelques exercices prospectifs de lignes nouvelles. Si la grande vitesse doit permettre de relier les grandes métropoles à $320 \mathrm{~km} / \mathrm{h}$ et si le réseau classique modernisé et fiable doit irriguer prioritairement l'échelon intrarégional, il reste que « d'autres services doivent pouvoir s'envisager autour des $200 \mathrm{~km} / \mathrm{h}$ à $220 \mathrm{~km} / \mathrm{h}$, avec des matériels nouveaux, confortables, rapides, à partir des infrastructures existantes » [12] Ce cran de vitesse manquant pourrait marquer une étape nouvelle de la doctrine ferroviaire portée par la planification nationale. Elle s'inscrit dans une évolution du rapport vitesse/territoire qui peut être précisée autour d'un exemple régional précis.

\section{De la dette historique au projet LNPN : l'évolution du critère vitesse dans la planification ferroviaire normande}

À travers l'exemple des territoires institutionnels normands récemment débattus [13], il s'agit d'apprécier la mobilisation du critère de la vitesse dans les exercices de prospective et de planification territoriale. Cet article ne traite donc pas de l'effet territorial de la grande vitesse débat complexe et controversé alimenté par des travaux académiques et institutionnels de grande qualité $[14 ; 6 ; 15 ; 16 ; 17 \ldots]$ -, mais bien de l'utilisation du critère vitesse dans les débats, les documents et plus globalement dans l'exercice d'aménagement du territoire.

Notre analyse prend en compte l'espace normand, sans ligne à grande vitesse, mais objet de nombreux débats et procédures de débats publics sur cette question, en ce qu'il constitue un exemple heuristique. Cette analyse est introduite par un rapide cadrage historique de l'évolution de la vitesse ferroviaire en Normandie. Elle s'attache d'abord à décrypter ce binôme vitesse-territoire autour de la procédure de débat public associée au projet de ligne ferroviaire Paris-Normandie (2011-2012). La lecture par le niveau national d'un axe Seine a ainsi inscrit la question de la relation entre le Grand-Paris et l'espace normand à l'agenda prospectif. Elle permet de cerner la mobilisation du critère de la grande vitesse pour proposer une lecture spécifique du territoire. L'analyse porte ensuite sur l'évolution du référentiel de la grande vitesse dans la planification. Au-delà du débat médiatisé sur l'axe Seine, la prise en compte de l'évolution du projet de Ligne nouvelle Paris Normandie (LNPN) - de la grande vitesse à la ligne nouvelle - éclaire le rapport nouveau entre vitesse, accessibilité et territoires. Cette évolution traduit d'une part une modification interne au gestionnaire d'infrastructures de son référentiel d'évaluation des grandes infrastructures, et annonce d'autre part une période de réflexion des collectivités territoriales sur le rapport entre vitesse et territoires. Ce dernier point de notre analyse permet d'identifier ces figures territoriales opposées repérables dans les débats d'aménagement en cours, débats territoriaux structurés par cette question de la vitesse ferroviaire.

\section{« La dette historique » ou le progressif éloignement par la grande vitesse du réseau normand}

Une histoire de la vitesse ferroviaire dans l'espace normand témoigne d'une trajectoire non linéaire de ces gains de temps sur les lignes historiques. Cette histoire peut être segmentée en deux temps. Du succès inattendu de la ligne Paris-Rouen (inaugurée en 1843, prolongée vers Le Havre et Dieppe en 1847, puis vers Caen en 1858) à l'électrification des lignes dans les années 1960 pour la partie Paris-Le Havre (plus tardivement pour la partie Paris-Caen au début des années 1990), les lignes normandes ont longtemps constitué un axe prioritaire de la planification ferroviaire progressivement nationale. L'augmentation continue de la vitesse sur l'axe Paris-Normandie situe ainsi les trois métropoles normandes, au début des années 1970, dans une situation élevée d'accessibilité au réseau national étoilé. 


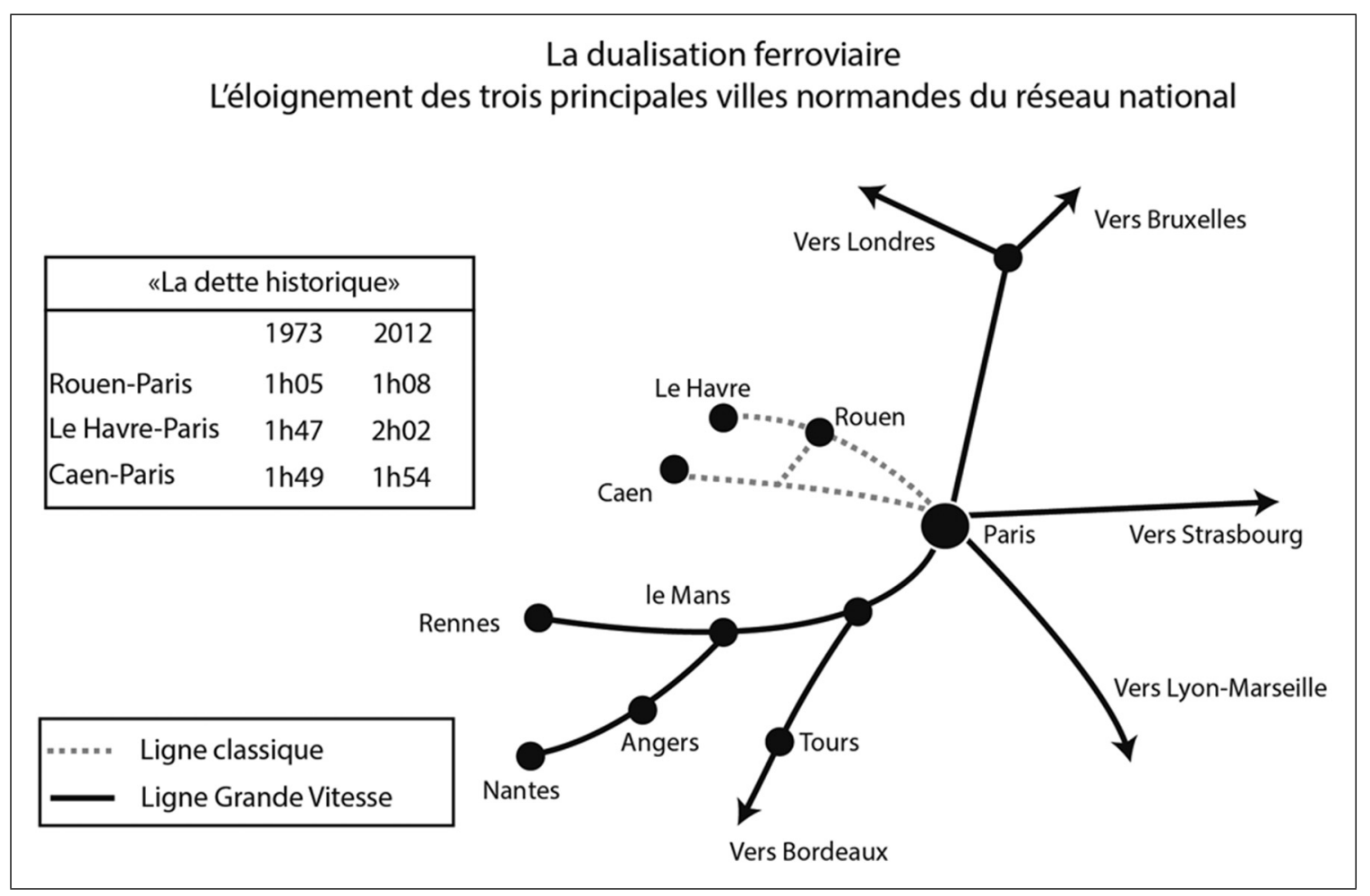

Fig. 1 La dualisation ferroviaire des trois principales villes normandes

Cette accessibilité va ensuite se détériorer, produisant une forme d'éloignement progressif des lignes normandes du réseau national. À cette accessibilité ici représentée sous le seul prisme de la vitesse, il faudrait bien sûr ajouter les fréquence, capacité, et régularité - ajouts qui amplifieraient cette image d'un éloignement ferroviaire. Ce recul, lié en grande partie au développement des lignes à grande vitesse sur le réseau français dans les années 1980 et 1990, témoigne d'une hiérarchisation nouvelle du réseau français. L'espace normand, paradoxalement trop proche de l'étoile parisienne pour être concerné par cette unification du territoire français par la vitesse, s'éloigne. Le désengagement financier du réseau secondaire, corolaire de la concentration des investissements sur la grande vitesse, se traduit par une augmentation des temps de parcours particulièrement significative sur l'axe Seine (figure 1). Le choix routier (généralisation des autoroutes) conjugué à cette nouvelle équation ferroviaire (priorité à la grande vitesse et aux trains de banlieue franciliens) dégrade l'accessibilité ferroviaire (temps de parcours, régularité). Cette trajectoire, bien sûr ici résumée de façon trop simpliste ${ }^{1}$, témoigne d'une mise à l'écart par la vitesse de l'espace normand. Ce constat

\footnotetext{
${ }^{1}$ Cette trajectoire historique dont nous résumons en quelques lignes la tonalité a été finement étudiée dans le cadre de deux mémoires universitaires récents (Université Paris 1, master aménagement et urbanisme), l'un portant sur l'interrégionalité ferroviaire dans l'espace
}

est d'autant plus fort que le démaillage ferroviaire du réseau national (36 $000 \mathrm{~km}$ de voies exploitées en 1970, 29000 en 2010) est important dans l'espace normand. À l'écart de la grande vitesse, desservies par un réseau secondaire en phase de contraction et non prioritaire dans les investissements, les trois métropoles normandes se retrouvent (du point de vue ferroviaire) éloignées des standards devenus classiques du réseau national, et ce à différentes échelles (relation Normandie-Paris, relation Normandie-Grand-Ouest, relations intra-normandes). Cet éloignement territorial par la grande vitesse est largement mesuré et même reconnu par l'opérateur ferroviaire. La déclaration au Havre en 2010 de Guillaume Pepy ${ }^{2}$, directeur de la SNCF, faisant étant d'une « sorte de dette vis-à-vis de la Normandie » et d'un « enclavement ferroviaire

normand [18], l'autre sur la géohistoire de l'axe Seine [19]. Cf. bibliographie.

2 « La SNCF a une sorte de dette vis-à-vis de la Normandie victime d'un effet de ciseau. D'un côté la fréquentation des lignes normandes est croissante, de l'autre, les performances sont décroissantes. Les trains mettent de plus en plus de temps et sont de moins en moins à l'heure. Nous sommes mal à l'aise avec cette dette que nous devons réparer [...]. Voilà vingt ans que l'on parle du désenclavement ferroviaire normand. Maintenant nous n'avons plus le droit d'échouer. Toutes les gares parisiennes ont connu la révolution de la grande vitesse, toutes sauf une ; Saint-Lazare. Son réseau date de 40 ans : il n'a pas bénéficié de l'effet TGV », Guillaume Pepy, Le Havre, 4 mai 2010. 
normand » en atteste. Elle s'inscrit d'ailleurs dans une réflexion renouvelée du rapport vitesse-territoire dans l'espace normand, actée dans les exercices récents de planification. La dégradation de l'accessibilité ferroviaire des deux régions normandes depuis 1972 est prise en compte dans la planification nationale des infrastructures de transport. Depuis le Schéma directeur national des liaisons ferroviaires à grande vitesse de 1991 jusqu'au récent projet de Schéma national des infrastructures de transport (SNIT) arbitré par la Commission 21, des liaisons dites «d'aménagement du territoire vers la Normandie » sont inscrites à chaque échéance de planification [18]. Mais c'est bien le projet récent de LNPN, lui-même composante d'une réflexion plus générale sur l'axe Seine, qui marque une relance de la réflexion vitesse-territoire dans l'espace normand.

\section{De la Très grande vitesse à la Ligne nouvelle Paris-Normandie}

Comme tout projet d'infrastructure de transport, l'idée d'une ligne à grande vitesse reliant Paris et la Normandie est une " réponse à un besoin formalisé par un acteur déterminant historiquement » [7]. Nicolas Sarkozy, dans son discours au Havre du 16 juillet 2009, lui-même inscrit dans l'exercice prospectif dit du " Grand Paris », définissait le projet LNPN comme " un transport rapide, un TGV, qui reliera Paris au Havre par Mantes et Rouen en une heure et quart et qui profitera également aux deux régions Normandie. Cette liaison à grande vitesse sera l'un des axes majeurs du Grand Paris ». Nicolas Sarkozy prolonge ainsi son discours d'avril 2009 où il préconisait de mettre Le Havre à $1 \mathrm{~h}$ de Paris, sans évoquer un passage par Rouen et Mantes, avec pour objectif de corriger une erreur géographique : «Paris est une ville-monde » qui mérite d'avoir une ouverture sur la mer. Ce deuxième discours de juillet traduit déjà un recalibrage vers une réponse aux besoins : 15 minutes de plus, mais un passage par Rouen et Mantes, dont on connaît l'étroitesse des relations avec Paris. La grande vitesse devient, dans le discours, l'outil principal d'un Grand Paris jusqu'à la mer ${ }^{3}$ esquissé par la proposition initiale d'une relation en $1 \mathrm{~h}$ entre Paris et Le Havre apportée par l'architecte Antoine Grumbach dans le cadre de l'Atelier du Grand Paris puis prolongé dans l'exercice prospectif notamment par les agences d'urbanisme et le milieu consulaire. La production d'une étude commune par 6 agences d'urbanisme (L'Axe Seine : une vision partagée)

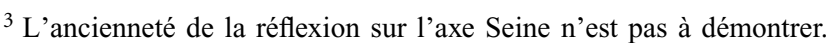
Anecdote éclairante, la devise de la compagnie Lafitte, concessionnaire de la ligne Paris-Le Havre en 1847 - Sic Lutetia portus (c'est par là que Paris devient port de mer) - témoigne de cette ancienneté.
}

publiée en mai 2011 ou encore le rapport Attali commandité par les Chambres de commerce et d'industrie du Havre, Fécamp-Bolbec et du Pays d'Auge publié en mai 2010 (Paris et la mer. La Seine est capitale) [20] témoigne du rôle structurant assigné à cette ligne dans la mise en place d'une interaction nouvelle entre l'espace normand et la région francilienne.

Ce discours politique initial n'a pas résisté à l'examen mené par le gestionnaire de l'infrastructure quant à la pertinence de desservir la Normandie avec une ligne à grande vitesse. Au-delà de la demande politique immédiate, la première phase de recueil des besoins et fonctionnalités du projet (associée à un recueil des fonctionnalités souhaitées par les acteurs du territoire, de leur hiérarchisation et des critères d'analyse des scénarios) par RFF, entre novembre 2009 et juin 2010, a constitué un préalable à l'analyse du projet en termes technique, environnemental et socio-économique. La forte mobilisation des acteurs a autorisé, sur ce projet, la mise en place d'un groupe de travail rassemblant les trois régions concernées, les services déconcentrés de l'État, la Datar et RFF complétés d'un important investissement des collectivités territoriales. Trente-six institutions ont finalement contribué à ce groupe de travail dont les conclusions confirment un consensus sur la nécessité d'une part d'amélioration des temps de parcours, des fréquences, des ponctualités, du confort et d'autre part de la structuration d'un réseau des villes normandes et de la conception d'un système de transport couvrant une multiplicité de besoins. Certes des divergences sur le niveau de service à atteindre et la crainte de l'amplification de fragmentations territoriales dues à cette ligne sont exprimées, mais sans contredire un consensus territorial général. Cette étude du contexte territorial, en juin 2011, confirme la décision de ne pas avoir recours à une LGV ferroviaire pour ce projet d'infrastructure. Les collectivités attendent du projet de ligne nouvelle qu'il améliore significativement (en fréquence et en temps de parcours) la liaison directe entre Paris et les trois agglomérations de Rouen, Le Havre et Caen (Paris-Rouen centre en 45 minutes, Paris-Le Havre et Paris-Caen en $1 \mathrm{~h} \mathrm{15)}$. Si ces objectifs de temps de parcours ne sont accessibles qu'à la condition de réaliser des sections de ligne nouvelle, la grande vitesse $(320 \mathrm{~km} / \mathrm{h})$ et le coût associé ne sont ici guère utiles. Dès lors, le débat public, qui débute en octobre 2011, propose «d'étudier une liaison nouvelle reliant Paris au Havre en 1 h 15 par Mantes et Rouen et qui profitera aux deux régions normandes. ». Le terme de liaison nouvelle signale la fin d'un projet de LGV « ralenti » au prisme de la réalité financière, des attentes territoriales et de la configuration géographique de la ligne (une distance courte). Elle signale aussi un changement de lecture pour le gestionnaire de l'infrastructure nationale qui doit développer 
un référentiel spécifique sur un cran manquant de la vitesse pour des mobilités quotidiennes sur des distances moyennes.

La lecture des cahiers d'acteurs sur cette période du débat public laisse apparaître un deuil difficile de la LGV annoncée par Nicolas Sarkozy dans son discours du Havre. Dans le débat public [21], les termes de TGV et de LGV ont longtemps été préférés à celui de « ligne nouvelle » par les acteurs territoriaux ${ }^{4}$. Ces «quatre pages » s'apparentant à une promotion des territoires, de la grande vitesse et de la combinaison parfaite des deux, sont éloquents. Mais la différence de presque $100 \mathrm{~km} / \mathrm{h}$ qu'il y a entre une LGV et la vitesse proposée pour la LNPN n'érode pas, pour la quasi-totalité des acteurs ayant déposé un cahier au débat public, une extrême motivation en faveur de ce projet considéré par exemple comme « enjeu vital ( (communauté de Bernay), d' « accélérateur économique » (communauté urbaine de Cherbourg), d'outil « du désenclavement régional » (syndicat mixte du Pays Dieppois) ou encore de « locomotive pour le développement du territoire » (syndicat mixte SCOT sud du pays d'Auge). La Communauté urbaine de Mantes en Yvelines définit clairement cette distinction entre la vitesse TGV et la vitesse Ligne nouvelle ${ }^{5}$ et, sans l'exprimer tous de la même façon, les acteurs intègrent alors cette réflexion sur un nouveau cran de vitesse. Au-delà du consensus général, ce critère de la vitesse (et ses arbitrages) introduit un rapport renouvelé de la ligne ferroviaire au territoire, c'est-à-dire une utilisation par les collectivités, les acteurs divers du débat public et par le gestionnaire de l'infrastructure nationale de ce critère pour appuyer des lectures du territoire situées, et donc partiellement opposées.

\section{La vitesse, outil de lecture territoriale : deux figures opposées ?}

\section{L'axe Seine ou comment positionner le $Y$, une question territoriale}

Chaque acteur mobilise logiquement le critère «vitesse » au prisme de son propre projet territorial. Cette remarque peut être triviale, mais l'analyse du débat public et des projets présentés témoigne, au-delà du consensus véritable

\footnotetext{
${ }^{4}$ Un constat d'ailleurs relevé dans le rapport de synthèse du président de la commission de débat public : "Vu les vitesses envisagées, il ne s'agit donc plus, comme il avait été initialement prévu d'une ligne à grande vitesse, ce que les participants aux réunions publiques semblent parfois avoir oublié » (mars 2012).

5 « La CAMY prend acte que la Ligne Nouvelle Paris-Normandie ne sera pas une ligne TGV « classique » mais bien une réponse adaptée et novatrice, en matériels et en dessertes, à des objectifs d'aménagement du territoire et de développement économique ».
}

sur la LNPN, de deux lectures partiellement opposées de cette relation vitesse-territoire dans l'espace normand. Cette opposition est mise à jour par l'analyse des trois projets présentés au débat public par RFF qui, assumant tous les trois des gains de vitesse importants entre l'Ile-de-France et les pôles de l'espace normand (une logique axiale) proposent par contre des rapprochements différenciés entre les trois métropoles normandes (le rapprochement intra-régional). Positionner le Y (la relation entre Paris, Rouen, Le Havre et Caen) revient finalement à assumer des lectures différentes de la relation axe Seine-territoires. Ce débat public sur la LNPN a été organisé du 3 octobre 2011 au 3 février 2012. Il porte sur deux parties distinctes, l'une consacrée au barreau Paris-Mantes avec l'objectif de séparer les trains franciliens et les trains normands (4 scénarios proposés) et l'autre sur la desserte normande et en particulier sur le point de bifurcation entre la ligne qui part vers Le Havre et celle qui se dirige vers la Basse-Normandie (3 scénarios proposés + un quatrième introduit en cours de débat). Les choix de la bifurcation (Louviers pour le scénario A, Évreux pour le scénario B, Pont-Audemer pour le scénario $C$ ) assument bien l'objectif de réduction des temps de parcours et d'amélioration des dessertes (fréquence, ponctualité) entre les destinations normandes, mais proposent des variations importantes de ces gains de parcours. Sans décrire l'ensemble des gains présentés par RFF dans une matrice incluant l'ensemble des villes normandes (étude d'optimisation du projet, mars 2012), il importe ici de signaler que ces scénarios portent ainsi deux visions : l'une plus favorable au rapprochement des principales villes normandes (le point de bifurcation à Louviers) et l'autre plus ancrée sur la structuration axiale de la relation Ile-de-France-Normandie (un point de bifurcation à Évreux, proche de Mantes). Le scénario C, le plus proche de l'idée d'un pôle normand métropolitain (rapprochement de Caen-Rouen-Le Havre) connecté à l'Ile-de-France est, quant à lui, hors de portée financière et difficilement environnementalo-compatible (traversée de l'estuaire). Les débats sur ce point de bifurcation ont été prolongés par l'introduction d'un scenario mixte A-B permettant d'assurer la relation Rouen-Caen en moins d'une heure via le passage par Évreux (gare nouvelle) en limitant le kilométrage de nouveaux tronçons (coût légèrement inférieur aux scénarios $A$ et $B)$.

Mesurer la position précise de chaque collectivité et de chaque acteur du débat public par rapport à ces scénarios est hors de portée, mais la lecture des cahiers d'acteurs et des synthèses du débat public montre un positionnement différent des parties prenantes sur cette question de la bifurcation. S'il existe un consensus sur le désengorgement de la partie francilienne du réseau et sur son prolongement vers les pôles normands (profitable à tous) identifiable par 


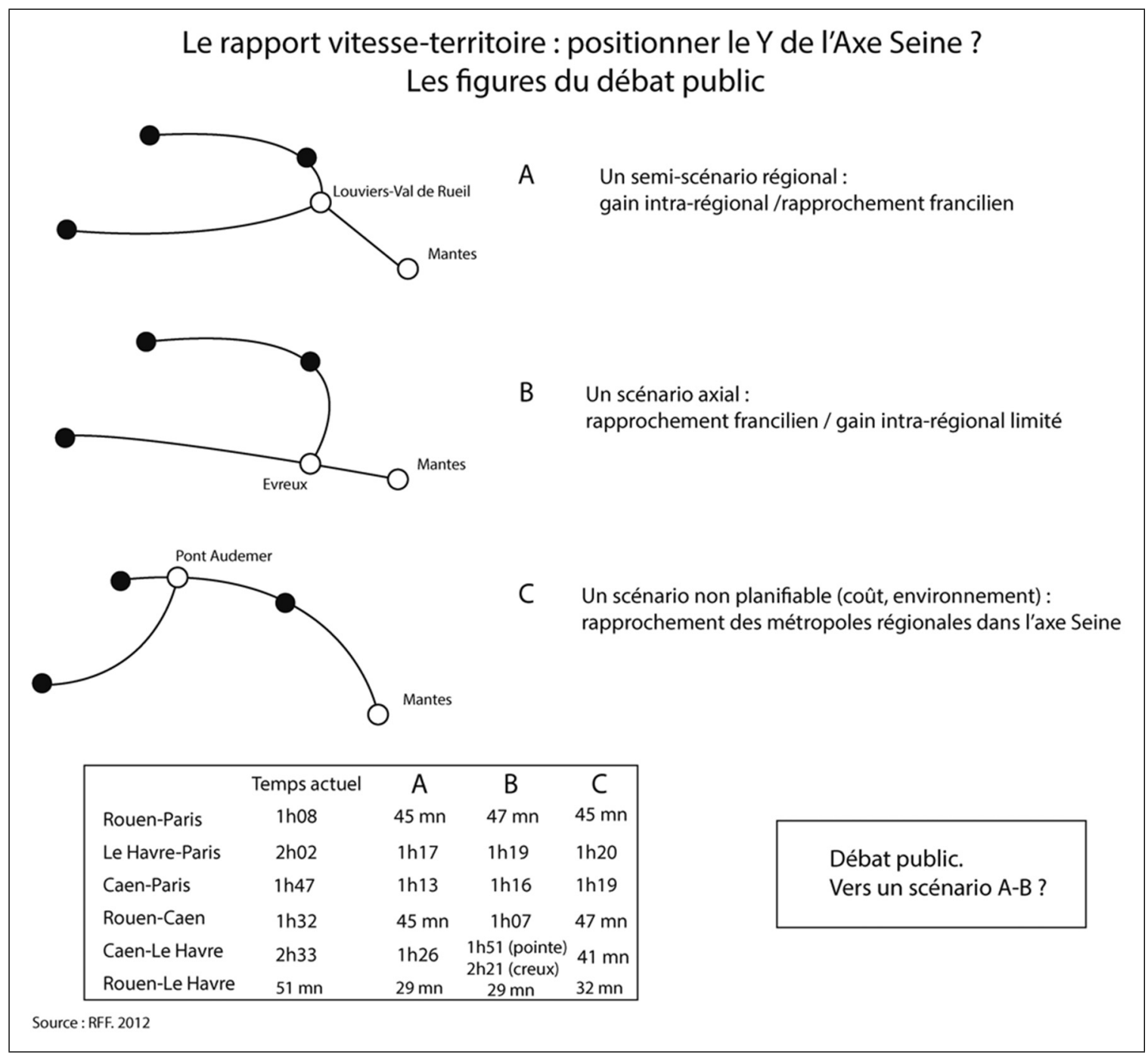

Fig. 2 Positionner le Y de l'Axe Seine

exemple dans le cahier d'acteurs des collectivités de l'axe Seine (cahier $\mathrm{n}^{\circ} 79$, janvier $2012^{6}$ ), des prises de position variées sur les scénarios sont identifiables. Les acteurs de la Basse-Normandie, le Conseil Régional en tête, sont ainsi favorables au scénario A (rapprochement avec Rouen et Paris), voire au scénario $\mathrm{AB}$, mais avec un appel pour prolonger l'effort au-delà de Caen - jusqu'à Saint-Lô et Cherbourg notamment (bilan du débat public, mars 2012). L'Union pour la région Normande prend logiquement position pour le scénario $\mathrm{C}$ dans un cahier d'acteurs $\left(\mathrm{n}^{\circ} 27\right.$,

\footnotetext{
${ }^{6}$ « La réalisation d'une ligne nouvelle en $\mathrm{Y}$, comportant un tronc commun indispensable entre Paris et Mantes, et deux branches, respectivement vers Lisieux, Caen, Cherbourg, et Rouen, Le Havre, ainsi que les raccordements permettant de relier Rouen à Évreux rapidement, constitue à ce stade des études et des scénarios proposés par le maitre d'ouvrage, le meilleur compromis », Collectivités de l'axe Seine, janvier 2012.
}

novembre 2011) intitulé « solution normande et ambition maritime ». Les prises de positions du Conseil Général de l'Eure ou encore de la Chambre de métier et d'artisanat de l'Eure (cahier d'acteurs $\mathrm{n}^{\circ}$ 51, décembre 2011) sont, sans surprise, favorables au scénario B pointent la situation potentielle de carrefour entre les deux normandies d'Évreux et l'importance du lien Évreux-Rouen. Les acteurs du débat public à Vernon (Génération Vernon, cahier d'acteurs $n^{\circ} 87$, juillet 2012) ou de Bernay (association des usagers de la gare SNCF de Bernay, cahiers d'acteurs $\mathrm{n}^{\circ} 101$, janvier 2012 ; ville de Bernay, cahiers d'acteurs $n^{\circ} 31$, novembre 2011) valident également ce scénario $B$ dans une logique de rapprochement au barreau francilien. Ces prises de position sont complétées d'intérêts plus localisés (réflexion sur la nouvelle gare pour Rouen, réflexion sur la nécessité de libérer des sillons pour le fret ferroviaire au Havre). La vitesse ferroviaire, même inférieure au critère LGV, reste un 
outil d'une prospective mobilisée pour construire des projets territoriaux logiquement situés.

\section{L'arbitrage, un axe normand. . . francilien}

Trois ans après le débat public et après l'arbitrage de la Commission 21, il semble se dégager un choix de planification largement réévalué par rapport aux figures discutées pendant le débat public. Les arbitrages récents, conformes à l'idée de hiérarchisation en fonction des priorités de désaturation, tendent à statuer sur un axe d'abord dédié à la désaturation du barreau francilien (Paris-Mantes) et du nœud rouennais (nouvelle gare de Saint-Sever) en intégrant néanmoins la problématique Mantes-Évreux. Ils se traduisent en gains de temps sur l'axe Seine Paris-Rouen-Le Havre et sur la relation Paris-Caen, mais ne remplissent pas les objectifs initiaux de rapprochement entre les trois principales villes normandes. Le gain de vitesse reste, dans cette logique, un outil radial (Paris-Normandie) et pose la question du maintien d'une fracture régionale ferroviaire impulsée par le dualisme intramodal signalé en introduction. Certes, l'accessibilité ne peut pas se résumer à cette mise en relation ferroviaire et le caractère structurant de ces axes a été largement relativisé dans la période récente. Mais cette mobilisation autour de la grande vitesse puis d'un nouveau cran de la vitesse en Normandie a, au final, débouché sur un projet centré sur la définition des temps de parcours en fonction des besoins du quotidien, sur la fréquence et sur la qualité pour l'accès au nœud parisien, complété d'une prospective intra-métropolitaine sur les quartiers de gare (Rouen notamment). Il reste que cette histoire récente du débat public et des arbitrages postérieurs témoigne de l'évolution de ce référentiel «vitesse » dans la prospective territoriale, c'est-à-dire de cette transition introduite par la hiérarchisation financière de la grande vitesse subordonnée à l'estimation des besoins du territoire.

\section{Conclusion : nouveau référentiel d'évaluation, nouvelles vitesses ferroviaires?}

L'objectif de ce travail était d'apprécier, autour d'un exemple précis, l'utilisation du critère de la vitesse ferroviaire dans la planification et la prospective territoriales. Différents éléments valident l'évolution du rapport vitesse/politiques/infrastructures. Le premier élément tient à l'émergence d'une nouvelle lecture ferroviaire basée sur la nécessité de réévaluer et de moderniser le réseau existant. Ce tournant, accéléré par la publication de l'expertise indépendante apportée par l'École Polytechnique de Lausanne sur le réseau ferroviaire français, acté par
RFF dans son Grand plan de modernisation du réseau ferroviaire est consacré par le rapport de la Commission 21. Du schéma national d'infrastructures (issu des lois Grenelle) au schéma de mobilité durable publié en 2013, une bascule de la lecture de l'infrastructure ferroviaire s'opère sur un temps très court. Cette nouvelle lecture se traduit-elle par une évolution générale du référentiel de la vitesse ? Annoncer la fin d'une période TGV serait bien sûr tout à fait abusif et le modèle ferroviaire français restera structuré sur un réseau LGV malgré le report, voire l'annulation, d'un certain nombre de projets de développement ${ }^{7}$. Il reste que l'émergence d'une réflexion sur un nouveau cran de la vitesse, en négociation entre RFF et les collectivités territoriales nous semble importante à relever. Ce nouveau rapport entre vitesses et territoires reste évidemment à évaluer au prisme des investissements réels à venir. Mais la publication du rapport de la Commission Mobilité 21 en juin 2013 marque une prise de conscience de l'impossibilité de généraliser l'accès à la grande vitesse pour tous les territoires. ${ }^{8}$ Du critère unique de la LGV à une recherche d'adaptation entre technique ferroviaire et réalité territoriale, c'est au fond la question classique de l'adhérence entre réseau et territoires qui est ici posée. L'espace normand en est un bel exemple. Les conclusions du projet LNPN [22], avant même la sortie du rapport de la Commission 21, sont arrivées à un constat identique et proposent de s'intéresser à une vitesse ferroviaire inédite en France, oscillant entre $220 \mathrm{~km} / \mathrm{h}$ et $250 \mathrm{~km} / \mathrm{h}$. Ce projet met en avant la réalité du territoire normand (qui ne correspond pas aux canons du concept TGV) : distances courtes affaiblissant les gains potentiels, absence de la nécessité de concurrencer les liaisons aériennes... Ce projet témoigne de la recherche d'adéquation entre la ligne nouvelle et les besoins des territoires, adéquation actuellement arbitrée par les perspectives de financement. L'exemple de la LNPN est certes singulier, mais on peut néanmoins y ajouter l'exemple similaire de la Ligne nouvelle Provence Alpes Côte d'Azur [23] ou encore les réflexions actuelles sur la ligne Nîmes-Montpellier. On pourrait également, pour appuyer ce constat de l'émergence d'une réflexion nouvelle de la vitesse ferroviaire, faire référence au choix récent de l'entreprise ferroviaire allemande de ne plus construire de lignes à plus de $250 \mathrm{~km} / \mathrm{h}$ pour privilégier une augmentation du confort sur les lignes existantes (nouvelle gamme de

\footnotetext{
${ }^{7}$ Les projets Tours-Bordeaux, Bordeaux-Toulouse, MontpellierPerpignan restent ainsi basés sur le « design » classique de la ligne à $320 \mathrm{~km} / \mathrm{h}$, complétant ainsi le réseau TGV existant.

${ }^{8}$ « L'approche de la loi d'orientation et d'aménagement du territoire de 1995, qui consistait à proposer à tous les territoires un accès à l'autoroute et à la grande vitesse ferroviaire n'a pas résisté longtemps à un examen sérieux. », Mot du président [12]. Cette idée avait été pourtant reprise par le volet transport du Grenelle de l'environnement en 2007, proposant la construction de $2000 \mathrm{~km}$ de LGV.
} 
train en 2017) ou encore mentionner la situation délicate en Espagne pour des projets de développement de lignes grande vitesse (à l'arrêt, sans jeu de mots) au final très peu rentables $(3000 \mathrm{~km}$ de LGV pour seulement 16 millions de passagers). La vitesse, et surtout le coût de cette vitesse, sont ainsi récemment rediscutés. Cette évolution, émergente et largement financière, pourrait s'inspirer de la musicologie espérée par Paul Virilio, c'est-à-dire " cette sagesse et cette intelligence de l'accélération et de la décélération, qui sont les deux aspects du même mouvement ${ }^{9}$ ». Si la médiatisation du rapport de la Commission 21 a permis de diffuser cette nouvelle lecture, l'imaginaire sociopolitique autour de la grande vitesse ferroviaire est pour autant encore bien présent dans les discours territoriaux, accentué par une difficulté à conceptualiser cette vitesse intermédiaire pour les projets à venir.

\section{Bibliographie}

1. Veltz P (2013) Paris, France, Monde. Éditions de l'aube, La tour d'Aigues.

2. Dubois S (2010) TGV : un quart de siècle de bouleversements géoéconomiques et géopolitiques. Géoéconomie 52 : 89-104.

3. Varlet J (2009) Les réseaux de transport rapides. Du désenclavement interrégional aux dualismes intermodaux. In La France : aménager les territories. Armand Colin.

4. Zembri P (1997) L'émergence des réseaux ferroviaires régionaux en France : quand un territoire institutionnel modifié s'impose au territoire fonctionnel. Flux $29: 25-40$.

5. Rivier R, Putallaz Y (2005) Audit sur l'état du réseau ferroviaire national français. École Polytechnique Fédérale de Lausanne.

6. Delaplace M (2012) TGV, développement local et taille des villes : Une analyse en termes d'innovation de services. Revue d'Économie Régionale et Urbaine : 265-290.

7. Plassard F (2003) Transport et territoire. PREDIT, La documentation française.

8. DIACT (2009) Les effets de la grande vitesse ferroviaire sur l'aménagement et le développement des territoires, État de l'art et analyse bibliographique, Études Setec Organisation, Paris
9. Gallez C, Kaufmann V, Maksim H, Vincent S (2010) L'action publique face à la mobilité. Éditions l'Harmattan.

10. Offner JM (2006) Les plans de déplacements urbains. La Documentation française.

11. Subra P (2012) Géopolitique de l'aménagement du territoire. Armand Colin.

12. Commission Mobilité 21 (2013) Pour un schéma national de mobilité durable. Rapport au ministre chargé des transports, de la mer et de la pêche.

13. Brennetot $\mathrm{S}$ et al. (2012) La Normandie en débat. OREP editions.

14. Blanquart C, Delaplace M (2009) Innovations relationnelles, nouvelles offres de service et valorisation des nouvelles infrastructures de transport, Le cas de d'une plateforme multimodale et d'une desserte TGV. Cahiers Scientifiques des Transports $56: 63-86$.

15. Bazin S, Beckerich C, Delaplace M (2011) Les effets des dessertes ferroviaires à grande vitesse : des divergences d'objectifs qui limitent la porte stratégique d'accompagnement. Colloque AISRE-ASRDLF 2011

16. Mignerey P (2013) Les effets territoriaux de la grande vitesse en France. Collection « territoire en mouvement », Datar.

17. Berion P (2007) L'évaluation socio-économique des infrastructures de transport : Enrichir les approches du développement territorial. Revue d'Économie Régionale et Urbaine : 651-676.

18. Gaudino P (2013) Interrégionalité ferroviaire et accessibilité au territoire. Le cas des Régions normandes. Mémoire de Master 1 aménagement et urbanisme, Université Paris 1 PanthéonSorbonne.

19. Goldberg J (2013) Deux projets ferroviaires vus par le prisme d'une approche géo-historique. L'exemple de l'Axe Seine et du corridor Québec-Windsor, mémoire de Master 2, aménagement et urbanisme, Université Paris 1 Panthéon-Sorbonne.

\section{Documentations institutionnelles principales}

20. Commission Attali (2010) Paris et la Mer. La Seine est capitale. Fayard.

21. Commission Nationale du Débat Public. (2012) Bilan du débat public Projet de ligne nouvelle Paris-Normandie.

22. Réseau Ferré de France (2013) Ligne Nouvelle Paris-Normandie. Étude d'optimisation du projet. http://www.lnpn.fr/

23. Réseau Ferré de France (2013) Ligne Nouvelle Provence-Alpes Côte d'Azur. Adapter la vitesse aux enjeux du territoire, synthèse des études de vitesse et temps de parcours.

\footnotetext{
${ }^{9}$ Evard J.L. Entretien avec Paul Virilio. Revue en ligne Conférence. Date inconnue.
} 